\title{
Analysis of plasmid genes by phylogenetic profiling and visualization of homology relationships using Blast2Network Matteo Brilli ${ }^{1,3}$, Alessio Mengoni ${ }^{1}$, Marco Fondi ${ }^{1}$, Marco Bazzicalupo ${ }^{1}$, Pietro Liò ${ }^{2}$ and Renato Fani*1
}

Address: ${ }^{1}$ Department of Evolutionary Biology, University of Florence, via Romana 17, I-50125 Florence, Italy, ${ }^{2}$ Computer Laboratory, University of Cambridge, 15 JJ Thompson Avenue, Cambridge, CB3 OFD, UK and ${ }^{3}$ Laboratoire de Biometrie et Biologie Evolutive, UMR CNRS 5558, Lyon, France

Email: Matteo Brilli - brilli@biomserv.univ-lyon1.fr; Alessio Mengoni - alessio.mengoni@unifi.it; Marco Fondi - marco.fondi@unifi.it; Marco Bazzicalupo - marco.bazzicalupo@unifi.it; Pietro Liò - pl219@cam.ac.uk; Renato Fani* - renato.fani@unifi.it

* Corresponding author

Published: 21 December 2008

BMC Bioinformatics 2008, 9:55I doi:|0.1|86/|47|-2105-9-55|
Received: 18 June 2008

Accepted: 21 December 2008

This article is available from: http://www.biomedcentral.com/I47I-2/05/9/55 I

(C) 2008 Brilli et al; licensee BioMed Central Ltd.

This is an Open Access article distributed under the terms of the Creative Commons Attribution License (http://creativecommons.org/licenses/by/2.0), which permits unrestricted use, distribution, and reproduction in any medium, provided the original work is properly cited.

\begin{abstract}
Background: Phylogenetic methods are well-established bioinformatic tools for sequence analysis, allowing to describe the non-independencies of sequences because of their common ancestor. However, the evolutionary profiles of bacterial genes are often complicated by hidden paralogy and extensive and/or (multiple) horizontal gene transfer (HGT) events which make bifurcating trees often inappropriate. In this context, plasmid sequences are paradigms of networklike relationships characterizing the evolution of prokaryotes. Actually, they can be transferred among different organisms allowing the dissemination of novel functions, thus playing a pivotal role in prokaryotic evolution. However, the study of their evolutionary dynamics is complicated by the absence of universally shared genes, a prerequisite for phylogenetic analyses.
\end{abstract}

Results: To overcome such limitations we developed a bioinformatic package, named Blast2Network (B2N), allowing the automatic phylogenetic profiling and the visualization of homology relationships in a large number of plasmid sequences. The software was applied to the study of 47 completely sequenced plasmids coming from Escherichia, Salmonella and Shigella spps.

Conclusion: The tools implemented by B2N allow to describe and visualize in a new way some of the evolutionary features of plasmid molecules of Enterobacteriaceae; in particular it helped to shed some light on the complex history of Escherichia, Salmonella and Shigella plasmids and to focus on possible roles of unannotated proteins.

The proposed methodology is general enough to be used for comparative genomic analyses of bacteria.

\section{Background}

Despite the huge amount of available sequences, few papers reported comparative analyses of entire plasmids with the aim of a complete classification of the functions they code for [1-4], and none considered all the sequences coming from entire genera or more inclusive taxonomic groups. 
Nevertheless, plasmids are extremely important in microbial evolution, because they can be transferred between organisms, representing natural vectors for the transfer of genes and functions they code for $[[5,6]$ and references therein]. In medical epidemiology and microbial ecology plasmids are thoroughly investigated because they often carry genes encoding adaptive traits such as antibiotic resistance, pathogenesis or the ability to exploit new environments or compounds [[7-9] and references therein].

While bacterial chromosomes show a relatively high conservation of their architecture, plasmid molecules are more variable concerning gene content and/or organization, even at short evolutionary distances. Indeed, plasmid genes can be considered to be under differential selection, while moving around the bacterial community. Moreover they have a dynamic structure, i.e. genes can be gained or lost from the plasmid molecule. Actually, the same plasmid can be hosted by different organisms inhabiting different environments (e.g.: $\mathrm{pH}$, temperature and chemical composition) and cohabiting with different genetic backgrounds. These factors may shape both the functional role(s) of the proteins, and the compositional features of plasmid DNA, such as GC or oligomers contents, some of the last being a very specific signature even at close phylogenetic distances [10].

Despite their key role in the microbial world, at least two main issues concerning plasmids remain poorly investigated: i) the function of proteins they code for (see Additional file 1, more than $25 \%$ of proteins do not have assigned COG) and ii) the evolutionary dynamics of plasmids including their importance in bacterial evolution [11].

This latter point is often analyzed using phylogenetic methods that make use of rigorous statistical approaches to model the evolution of sequences (such as Maximum Likelihood or Bayesian inference). However, such methods are of restricted use in the case of plasmid molecules: they are computationally expensive when thousands of amino acid or nucleotide sequences are analyzed, and, moreover, require a set of homologous and universally shared sequences, that could be unavailable when studying plasmids.

To overcome these limitations we have developed a bioinformatic package (Blast2Network, B2N) having three main aims:

1) to reconstruct the evolutionary history of plasmids molecules by identifying those having the most similar gene content;

2) to assign a putative function to previously uncharacterized proteins. This task is fulfilled in two ways: by means of sequence similarity of unknown or hypothetical proteins to known ones and through a phylogenetic profiling approach. In this case the function of a protein is inferred by observing co-occurrence patterns. This is based on the idea that proteins involved in the same metabolic process or macromolecular complex tend to be maintained (or lost) together and that proteins which often occur together are likely to be functionally linked [12].

3) to provide an immediate visualization of the similarities existing among sequences. In fact, one of the outputs of the program is a network of sequence similarities in a format readable by the visualization software Visone http://visone.info/.

To test the package, we focused the attention on plasmids harbored by members of the Enterobacteriaceae family of $\gamma$-Proteobacteria, which is one of the most studied divisions of bacteria and includes Escherichia, Shigella, and Salmonella genera, whose biomedical importance [13] has allowed to record a relatively high number of completely sequenced plasmids in a few species. Moreover, horizontal transfer of plasmids between them has been described [14], complicating the phylogenetic information on plasmids; lastly, several pathogenesis-associated phenotypes are plasmid-borne [15]. Consequently, the application of $\mathrm{B} 2 \mathrm{~N}$ to this dataset could allow to reveal the presence of relationships between known pathogenesis-associated proteins and those which have not been characterized yet.

\section{Methods}

\section{Description of the program}

The procedure implemented in $\mathrm{B} 2 \mathrm{~N}$ is schematically reported in Figure 1a, but several tasks can be performed separately because of the modular nature of our software. The main workflow starts from a file containing protein or nucleic acid sequences in standard NCBI fasta format. This is used as an input to gather information on source sequences from the NCBI website. Several files are automatically generated for reference along with the corresponding nucleotide sequences for both genes and source sequences (e.g. the genome or the plasmid encoding the proteins used as input). Input sequences are then screened one against each other using BLAST [16]. The resulting output is parsed in the form of an adjacency matrix that describes the global sequence similarities in the dataset where each entry $w_{i j}$ reflects the similarity existing between protein $i$ and $j$. The user is initially prompted to choose two different selection criteria for alignments: an E-value threshold and an alignment length cut-off; after setting these parameters, all alignments passing the selection criteria are inserted in the matrix. Moreover, the user can specify the nature of the similarity score to be used, i.e. identity percentage or bit score; the bit score can also be normalized using the score of the alignment of the query with itself obtaining a value which is normalized on the 
a)

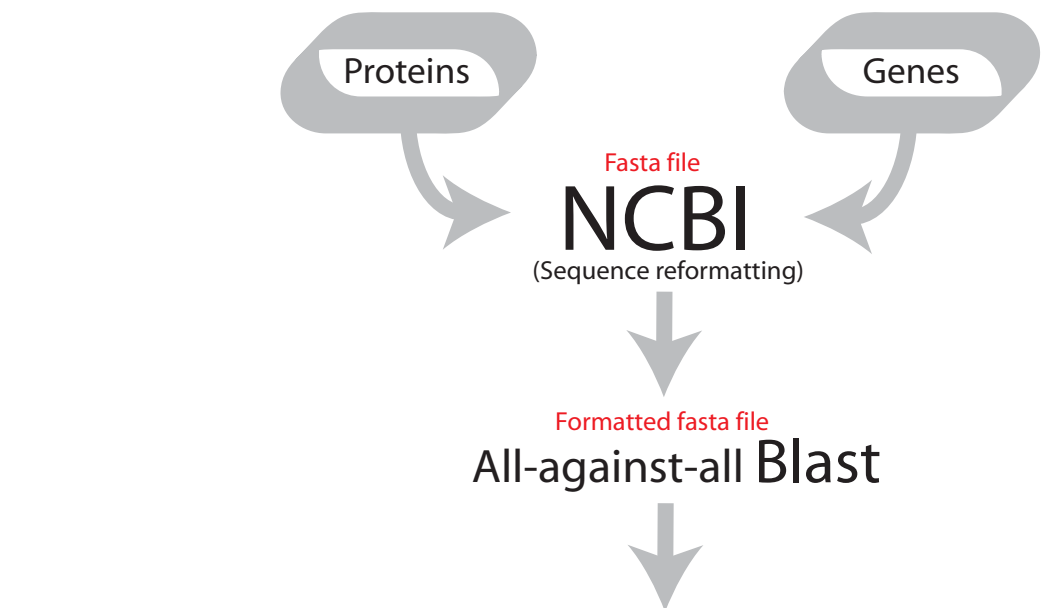

Adjacency matrix

Blast output file

\section{Phylogenetic profiling}

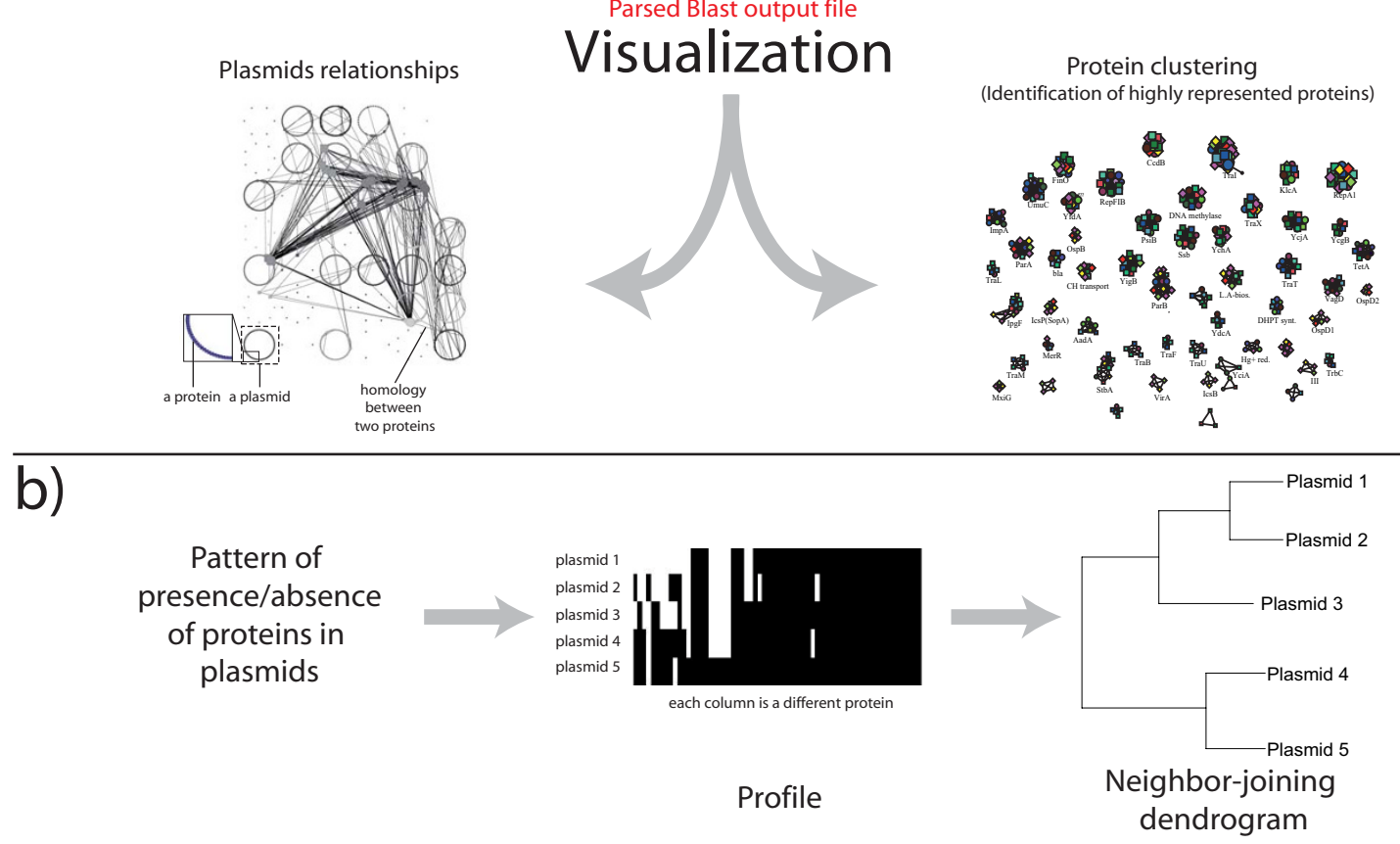

Figure I

B2N workflow and analysis. a) Scheme of the data workflow in B2N. The visualization is realized using Visone software. The input of each module (i.e. the output of the previous one) is shown in red fonts. b) Phylogenetic profiling of molecules in the dataset. Using the matrix of occurrence patterns, groups of proteins are identified at different threshold values. A new matrix is obtained composed of a row for each plasmid in the dataset and a number of columns corresponding to the number of groups in the network. Each entry $i, j$ of the matrix contains $I$ if at least one protein from plasmid $i$ is present in cluster $j, 0$ if no protein from plasmid $i$ is present cluster $j$. This matrix is used for calculating distances using the Jaccard metric and dendrogram construction. This analysis identifies those plasmids that contain similar proteins. By applying the same workflow in the second dimension of the phylogenetic profiles matrix, it is possible to find those protein clusters having similar occurrence patterns. 
alignment length. The weighted link values can be useful when comparing sequences from different species searching for those having the highest rate of horizontal transfer. This can be done in $\mathrm{B} 2 \mathrm{~N}$ specifying a distance matrix of house-keeping genes in Phylip format. The adjacency matrix obtained by parsing the BLAST output is the input for the phylogenetic profile method.

\section{Phylogenetic Profiling}

This approach allows the analysis of co-occurrence patterns, metabolic reconstruction and so on. In details, by taking as input the adjacency matrix storing the sequence similarity values, B2N produces a rectangular matrix (as described in the central part of Figure 1b) composed by all the plasmids under analysis (rows) and all the protein clusters (columns) identified through a depth-first search of the adjacency matrix. Each position of the phylogenetic profile matrix will be " 1 " in the case a given plasmid (row) possesses (at least) one protein in the corresponding protein cluster (column), whereas it is filled with " 0 " in the opposite case.

One of the commonly used metrics for binary data comparison is the Jaccard similarity coefficient. Given two vectors of phylogenetic profiles in binary form (A and B in this case, with $\mathrm{n}$ observations), the Jaccard coefficient is defined as the size of the intersection divided by the size of the union of the sample sets: $J(A, B)=|A \cap B| /|A \cup B|$. The 'Jaccard distance', which measures dissimilarity between sample sets, is obtained by dividing the difference of the sizes of the union and the intersection of two sets by the size of the union: $\mathrm{J}_{\delta}(\mathrm{A}, \mathrm{B})=|\mathrm{A} \cup \mathrm{B}|-|\mathrm{A} \cap \mathrm{B}| /$ $|A \cup B|=1-J(A, B)$.

The Jaccard coefficient is a useful measure of the overlap that the attributes of 'A' and 'B' share. Each attribute of 'A' and ' $\mathrm{B}$ ' can either be 0 ('absence') or 1 ('presence'). The total number of each combination of attributes for both 'A' and 'B' are specified as follows: $M_{11}\left(M_{00}\right)$ represents the total number of attributes where 'A' and ' $\mathrm{B}$ ' both have a value of $1(0) . M_{01}\left(M_{10}\right)$ represents the total number of attributes where the attribute of ' $\mathrm{A}$ ' is 0 (1) and the attribute of ' $\mathrm{B}$ ' is $1(0)$. Each attribute must fall into one of these four categories, meaning that their sum equals $n$. The Jaccard similarity coefficient is $\mathrm{J}=\mathrm{M}_{11} /\left(\mathrm{M}_{01}+\mathrm{M}_{10}+\right.$ $\mathrm{M}_{11}$ ). Blast2Network calculates the Jaccard distance for both dimensions of the phylogenetic profiles matrix, which corresponds to the distance between plasmids in term of shared genes, and the distance between occurrence patterns of clusters in plasmids. The Jaccard distance matrices are then used for the construction of two neighbor-joining dendrograms (Figure 1b). The first one describes similarities in gene content of the plasmids, the other one groups together those protein clusters with the most similar occurrence pattern within plasmids. Ran- dom permutations of the original data allows to compute the statistical significance of the Jaccard distances.

\section{Network construction}

$\mathrm{B} 2 \mathrm{~N}$ also outputs the BLAST post processing results as a network in Visone format http://visone.info/, a freely available software for network visualization and analysis. In doing so, it takes advantage of several information: the position and the color of the nodes (proteins) in the network correspond to the plasmid source, whereas the links indicate the existence of a given degree of sequence similarity between nodes. To reduce the dimensionality of the networks it is possible to use the Jaccard distance matrices to construct two hypergraphs where each plasmid or protein cluster, respectively, are collapsed to single nodes connected by edges whose values reflect the significance of the Jaccard distance calculated (see below and in Additional file 2).

\section{Additional tools}

B2N can include additional information in the network, assigning to each node a numerical (or binary) value which can be visualized in Visone as the size of the node; this node-associated value might be a compositional measure, such as the GC content and/or the codon adaptation index $[17,18]$ of the corresponding gene. To this purpose, $\mathrm{B} 2 \mathrm{~N}$ has two methods but the user can input its own list of values as a text file. The first built-in method writes node values corresponding to the GC content of a sequence, while the other one implements the dinucleotide analysis derived from [10] and [19], obtaining a composition-based dissimilarity index of a gene sequence with respect to the source plasmid (or genome). Considering each possible dinucleotide, say $x y$, and a gene $s, x y(s)=$ $\left(f_{x y(s)} / f_{x(s)} * f_{y(s)}\right)$. From this value the program obtains $(s, g)$ $=1 / 16^{*}\left|{ }_{x y(s)}{ }^{-} x y(g)\right|$ over all 16 dinucleotides, that is a measure of the compositional bias of a given sequence $(s)$ with respect to a reference sequence $(g)$ i.e. the genome or the entire plasmid. The can be used to detect genes that have been recently transferred and have since then maintained the compositional properties of the original plasmid.

\section{Sequence data source and software availability}

The dataset used in this work is composed by all the proteins encoded by the available completely sequenced plasmid sequences from Escherichia, Shigella, and Salmonella genera (Table 1). Complete plasmid sequences were downloaded from the NCBI $\mathrm{ftp}$ website $\mathrm{ftp}: / /$ ftp.ncbi.nih.gov/refseq/release/plasmid.

The software $\mathrm{B} 2 \mathrm{~N}$ with the user's manual can be directly requested to the authors and is also available as Additional file (Additional file 3). 
Table I: Plasmids analyzed

\begin{tabular}{|c|c|c|c|c|}
\hline Plasmid & Organism & Length (nt) & \# ORF & Accession Number \\
\hline R72I & Escherichia coli & 75582 & 91 & NC 002525 \\
\hline p9123 & Escherichia coli & 6222 & 8 & NC 005324 \\
\hline pCI5-la & Escherichia coli & 92353 & 100 & NC 005327 \\
\hline pCol-let & Escherichia coli & 5847 & 7 & NC 002487 \\
\hline PAPEC-O2-R & Escherichia coli & 101375 & 119 & NC 00667I \\
\hline pColK-K235 & Escherichia coli & 8318 & 7 & NC 00688I \\
\hline pRK2 & Escherichia coli & 5360 & 6 & NC $\quad 005970$ \\
\hline pECO29 & Escherichia coli & 3895 & 2 & NC 001537 \\
\hline CloDFI3 & Escherichia coli & 9957 & 8 & NC 002119 \\
\hline PBHRKI8 & Escherichia coli & 5721 & 4 & NC 005568 \\
\hline PBHRKI9 & Escherichia coli & 5721 & 4 & NC 005569 \\
\hline pFLI29 & Escherichia coli & 6464 & 4 & NC 005923 \\
\hline PAPEC-O2-ColV & Escherichia coli & $|8450|$ & 209 & NC 007675 \\
\hline pCoo & Escherichia coli & 98396 & 94 & NC 007635 \\
\hline pBI7I & Escherichia coli & 68817 & 80 & NC 002142 \\
\hline $\mathrm{pOII3}$ & Escherichia coli & 165548 & 155 & NC 007365 \\
\hline PLGI3 & Escherichia coli & 6293 & 7 & NC 005019 \\
\hline pIGALI & Escherichia coli & 8145 & 3 & NC 005248 \\
\hline pl658/97 & Escherichia coli & |2549| & 141 & NC 004998 \\
\hline PKLI & Escherichia coli KL4 & 1549 & 1 & NC 002145 \\
\hline $\mathrm{pO} 157$ & Escherichia coli OI57:H7 str. Sakai & 92077 & 184 & NC 002128 \\
\hline POSAKI & Escherichia coli OI57:H7 str. Sakai & 3306 & 3 & NC 002127 \\
\hline pSFDIO & Salmonella choleraesuis & 4091 & 6 & NC 003079 \\
\hline pOUIII3 & Salmonella enterica & 80156 & 89 & NC 007208 \\
\hline $\mathrm{pC}$ & Salmonella enterica serovar Enteritidis & 5269 & 4 & NC 003457 \\
\hline PBERT & Salmonella enterica subsp.enterica serovar Berta & 4656 & 9 & NC 001848 \\
\hline pKDSC50 & Salmonella enterica subsp. enterica serovar Choleraesuis & 49503 & 48 & NC 002638 \\
\hline cryptic_plasmid & Salmonella enterica subsp.enterica serovar Choleraesuis & 6066 & 7 & NC 005862 \\
\hline
\end{tabular}


Table I: Plasmids analyzed (Continued)

\begin{tabular}{|c|c|c|c|c|}
\hline pSCV50 & Salmonella enterica subsp. enterica serovar Choleraesuis & 49558 & 51 & NC 006855 \\
\hline pSCI38 & Salmonella enterica subsp.enterica serovar Choleraesuis & 138742 & 170 & NC 006856 \\
\hline $\mathrm{pHCMI}$ & Salmonella enterica subsp. enterica serovar Typhi str. СТ। 8 & 218160 & 235 & NC 003384 \\
\hline $\mathrm{pHCM} 2$ & Salmonella enterica subsp.enterica serovar Typhi str. CTI 8 & 106516 & 105 & NC 003385 \\
\hline $\mathrm{pP}$ & Salmonella enteritidis & 4301 & 3 & NC 003455 \\
\hline pK & Salmonella enteritidis & 4245 & 3 & NC 003456 \\
\hline $\mathrm{pB}$ & Salmonella enteritidis & 1983 & I & NC 005002 \\
\hline R27 & Salmonella typhi & $|8046|$ & 207 & NC 002305 \\
\hline pSCIOI & Salmonella typhimurium & 9263 & 6 & NC 002056 \\
\hline $\mathrm{R} 64$ & Salmonella typhimurium & 120826 & 135 & NC 005014 \\
\hline pU302S & Salmonella typhimurium & 3208 & 4 & NC 006815 \\
\hline pU302L & Salmonella typhimurium & 84514 & 103 & NC 006816 \\
\hline PSLT & Salmonella typhimurium & 93939 & 112 & NC 003277 \\
\hline pSB4_227 & Shigella boydii Sb227 & 126697 & 148 & NC 007608 \\
\hline pSDI_197 & Shigella dysenteriae SdI 97 & 182726 & 223 & NC 007607 \\
\hline pWR50I & Shigella flexneri & 221851 & 293 & NC 002698 \\
\hline pCP30I & Shigella flexneri 2a str. $30 \mathrm{I}$ & 221618 & 261 & NC 004851 \\
\hline Coljs & Shigella sonnei & 5210 & 3 & NC 002809 \\
\hline pSS_046 & Shigella sonnei Ss046 & 214396 & 238 & NC 007385 \\
\hline
\end{tabular}

The table lists the plasmids used in this work, their accession numbers, their host organism, their length, and the number of proteins their genes code for.

\section{Results and discussion}

Visual representation of sequence homology network B2N was used to study the relationships existing between homologous proteins from all the completely sequenced plasmids available from three $\gamma$-Proteobacterial genera: Escherichia, Shigella, Salmonella. The dataset contains a total of 3701 ORFs, from 47 different plasmids (Table 1). To our knowledge, no attempt was made to describe in a meta analysis the overall body of plasmid sequence data in these species. Figure 2 shows the graphical representation of two networks generated with $\mathrm{B} 2 \mathrm{~N}$ using protein sequences in our dataset and using an aminoacid sequence identity threshold of $90 \%$ or $100 \%$ (Figure 2 a and $2 \mathrm{~b}$ respectively, where the thresholds are particularly high and the number of plasmids reduced to 39 out of 47 for clarity purposes). Proteins from the same plasmid are circularly arranged around the same centre and share the same color; proteins from the same genus are represented by the same shape (Figure 2c). The networks, obtained choosing an E-value threshold of 0.0001 and a minimum alignment length of 70 residues, have been visualized using the software Visone. The size of the nodes is proportional to the number of links they have. The analysis of Figure 2 revealed that most plasmids are strongly connected to others, but there are also plasmids exhibiting just few connections (see the section Phylogenetic profiling).

Focusing on protein clusters instead of plasmids, we can arrange nodes in an uniform visualization, where nodes are clustered together if they directly or indirectly share at least one link (Figure 3, with a threshold of $40 \%$ identity). 


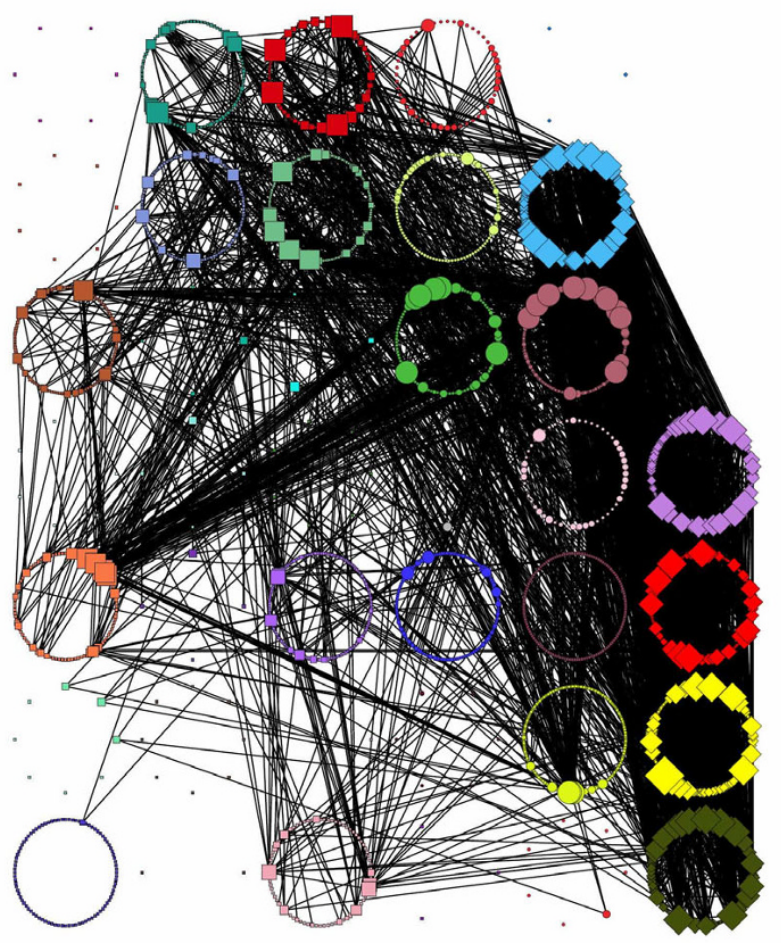

a

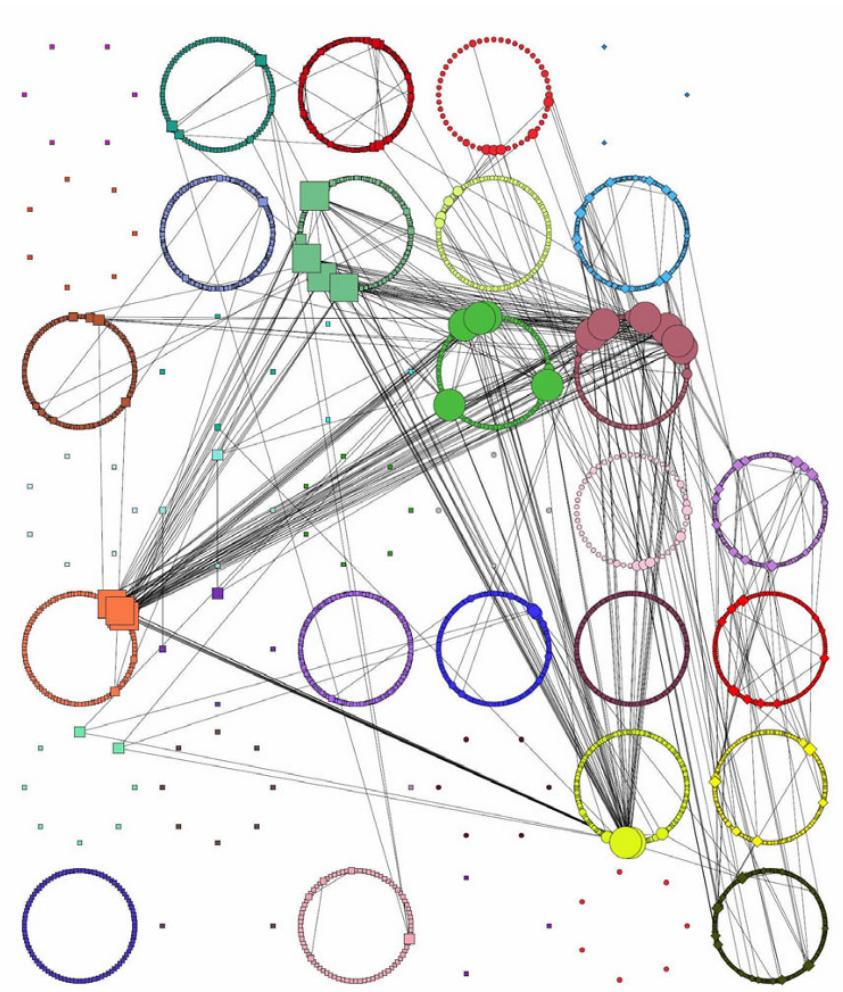

b

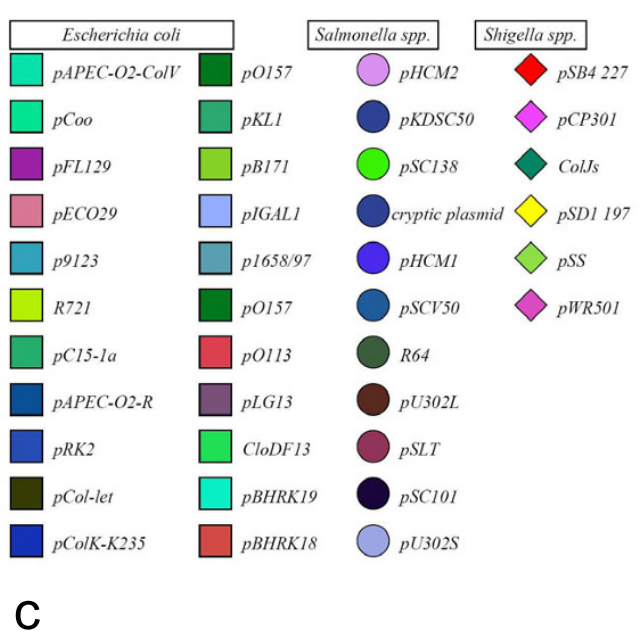

Figure 2

Plasmid homology networks. The output of B2N launched on the proteins encoded by 39 plasmids of three enterobacteria. Each protein in the dataset (see Table I) is arranged circularly with proteins from the same source plasmid; proteins from the same plasmid are shown the same colour. Links connecting different nodes represent alignments found by BLAST (length > 70 amino acids and E-value $<0.0001$ ); consequently they describe the relationships existing between plasmids with a $90 \%$ (a) or $100 \%$ (b) identity cut-off; c) graphical legend. Symbols: squares, circles, and diamonds represent E. coli, Salmonella and Shigella plasmid proteins, respectively. 
Quite interestingly, clustering of similar sequences at lower thresholds permits to assign a putative function to unknown or hypothetical proteins, and to discover the presence (if any) of functional classes or metabolic pathways that are more common in the network.

One of the problems faced with such complex data is the reduction of the dimensionality, so that important relationships can be more easily identified. Similarities in gene content between different plasmids can be better visualized by collapsing all the proteins belonging to the same plasmid in a single node. In this way a hypergraph is obtained where each node represents a single plasmid. The connection can be obtained from the plasmid vs plasmid Jaccard distance matrix or better, they can reflect the p-values matrix, so that each link in the hypergraph quantifies the significance of a given association between plasmids (showed in Additional file 2) and a simple hard thresholding allows changing the stringency for the inclusion of edges in the hypergraph.

\section{Network data analysis}

The analysis of the network data represented in Figures 2 and 3 revealed several interesting features of the relationships among the sequenced plasmids of the three genera under investigation:

1) Out of a total of 3701 proteins in the dataset, we found $1633(44 \%)$ and $2471(66.7 \%)$ isolated nodes at a threshold of $90 \%$ or $100 \%$ of identity for links, respectively (Figure $2 \mathrm{a}$ and $2 \mathrm{~b}$ ).

2) Most plasmids contain at least some gene coding for highly interconnected proteins; however, some of them (e.g. pRK2, ColJs Cjl, pLG13, CloDF13) exhibited only few connections. Hence, these plasmids share few genes with the other members of the dataset at these threshold levels. This, in turn, may suggest that they might have experienced less recombination events than others.

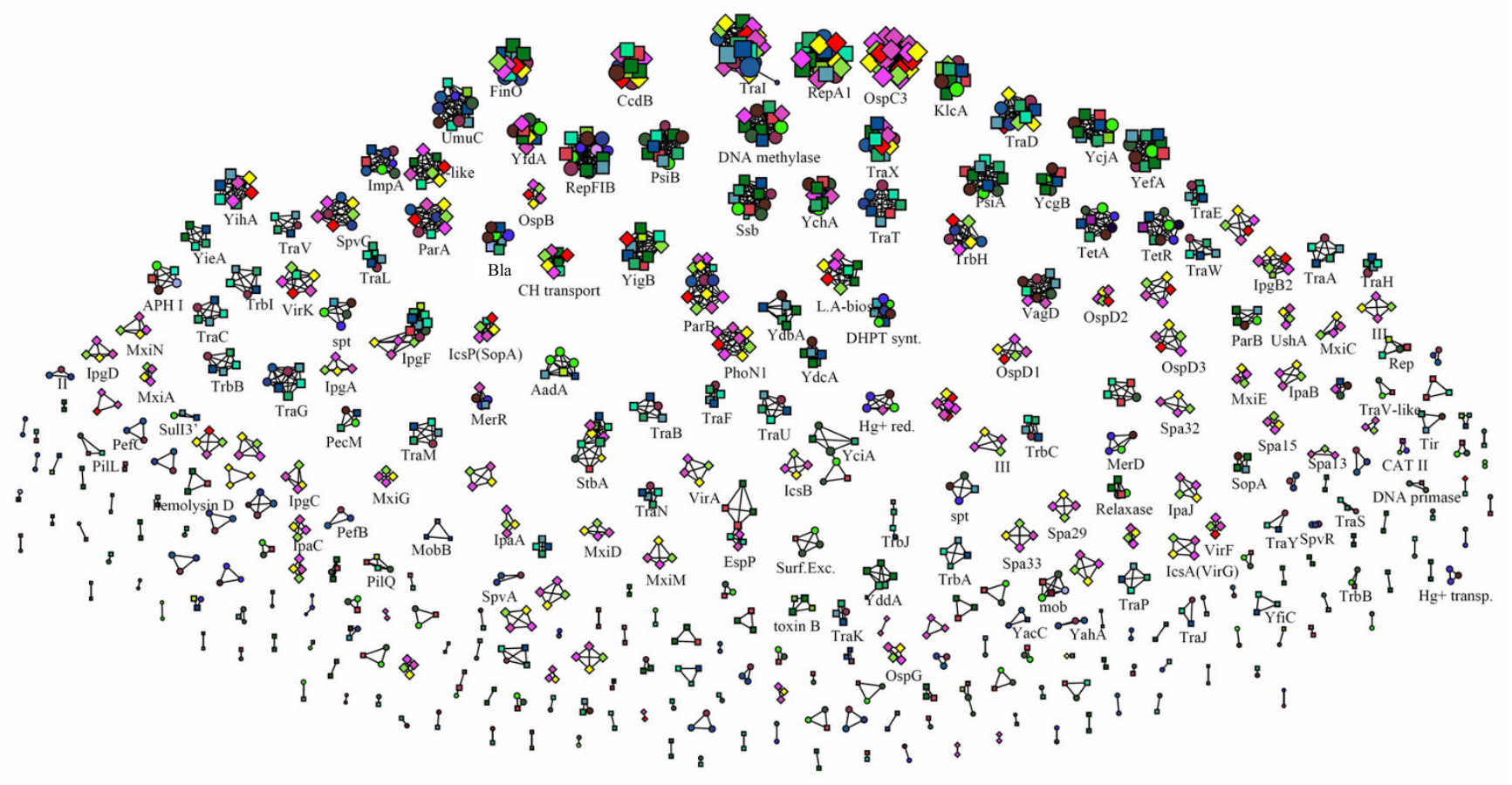

\section{Figure 3}

Uniform visualization of protein clustering. Uniform visualization of the similarity network for all of the $370 \mathrm{I}$ proteins, displayed using a threshold identity for links of $40 \%$ (a degree of amino acid sequence identity sufficiently high to cluster together proteins that should perform the same function, and also allowing a better defined separation of all the main protein clusters [29,30]). Groups of homologous proteins are separated, allowing the identification of proteins that very likely share an identical/similar function. The labels for some groups of proteins discussed in the text or very common are shown: KlcA, antirestriction protein involved in the broad-host range of IncP plasmids; FinO, RNA chaperone related to repression of sex pilus formation; $\mathrm{CcdB}$, protein involved in plasmid stability by killing bacteria that lose the plasmid during cell division; TetA and TetR, proteins responsible for resistance to tetracycline; Bla, $\beta$-lactamases; AadA, and DHPT synthase, proteins involved in resistance to aminoglycosides or sulfonamides, respectively. Tra and Trb, proteins requested for sex pilus formation; Mxi, Spa, Ipa, Ipg and Osp, proteins that are part of the type III secretion system. 
3) Several proteins (about $40 \%$ of all the connected nodes) were found to be mobile elements (transposases, IS and transposons -related sequences), representing the most highly connected proteins in the network.

4) As shown in Figure 3, proteins shared by Escherichia, Salmonella and Shigella plasmids included: a) the antirestriction protein KlcA involved in the broad-host range of IncP plasmids [20]; b) the RNA chaperone FinO, related to repression of sex pilus formation [21,22]; c) the $\mathrm{CcdB}$ protein, which is involved in plasmid stability by killing bacteria that lose the plasmid [23].

5) Several clusters were composed by proteins shared by Shigella spp. and Escherichia coli; this finding is in agreement with the notion that they are considered to belong to the same species [24]. Moreover, several proteins were shared only by E. coli and Salmonella plasmids, including: the genetic determinants for antibiotic resistance such as TetA and TetR [25], $\beta$-lactamases (Bla) $[25,27]$, genes for resistance to amino glycosides (AadA) and sulphonamides (DHPT synthase). A similar scenario was observed for sex pilus related proteins, such as Tra and Trb proteins: out of 22 different Tra groups, 21 contain proteins coming from E. coli and Salmonella, but 3 groups only (TraDI for DNA transport and TraX for pilin acetylation) have Shigella sequences. Likewise, out of 5 different Trb groups, we observed Shigella plasmid sequences in a single cluster (TrbH). Moreover, the proteins TraP, TrbA and TrbJ seem to be only present in plasmids from $E$. coli, while all the other sex pilus related proteins are shared with Salmonella. These data are in agreement with evidences for recent transfer of plasmid genes between enteroinvasive Escherichia and Salmonella $[26,27]$.

Concerning the pathogenesis-related genes, Shigella plasmids seem to have a specific set of these genes, comprising at least some of the proteins of the type III secretion system (TTS), e.g.: Mxi, Spa, Ipa, Ipg and Osp proteins.

Finally, on the overall observation it appeared that besides the closer phylogenetic relationships existing between E. coli and Shigella, plasmid content appeared more similar among E. coli and Salmonella for what is concerned with antibiotic resistance and sex pilus formation.

\section{Phylogenetic Profiling}

Data discussed in the previous paragraphs, that is which proteins join a given cluster, were stored by $\mathrm{B} 2 \mathrm{~N}$ into a text file, which represent the phylogenetic profile of the dataset used; this can be further used by the program to calculate two matrices storing the distances between profiles in the two dimensions (i.e. for plasmids and for proteins), as described in Methods. The corresponding neighbor-joining dendrograms, that describe the similar- ity in gene content of the plasmids and protein co-occurrence patterns are shown in Figure 4, Figure 5 and Additional file 4. Data reported in Figure 4 revealed that most of plasmids does not form tight clusters coherent with the taxonomic status of their respective host species (E. coli, Salmonella or Shigella). This finding suggests a complex evolutionary history of such plasmid replicons with massive horizontal transfer and gene rearrangements. In particular, plasmid pSFD10 from Salmonella grouped with two E. coli plasmids (pRK2 and pLG13).

A relevant exception is represented by five Shigella plasmids (pCP301, pSB4 227, pSD1 197, pWR501, and pSS 046) that form a unique clade (which, however, also includes pC plasmid from Salmonella enterica).

Figure 5 and Additional file 3 report the co-occurrence clustering for the protein dataset of the selected plasmids. In general, plasmids are believed to share very few common functions (mainly related to their replication and mobility), several accessory genes and a complex history of recombination events among either them or the host chromosome(s) [28]. Here, we actually show that most of the co-occurrence clusters are due to protein related to plasmid transfer (e.g. Trb and Tra proteins). Nevertheless, several clusters are present showing the co-occurrence of hypothetical proteins with proteins with predicted functions such as type II secretion proteins and pilins (BfpK), or with proteins involved in mobilization (MobA, MbkC) and virulence factors (IroN). These analyses may help in addressing experimental analyses for elucidating the functional role of these proteins.

\section{Conclusion}

In conclusion, we report that the tools implemented by $\mathrm{B} 2 \mathrm{~N}$ allow to describe and to visualize in a new way some of the evolutionary features of plasmid molecules of Enterobacteriaceae; the most important results obtained by $\mathrm{B} 2 \mathrm{~N}$ on the Enterobacteriaceae dataset are related to the possibility, by means of phylogenetic profiling and network relationships of proteins, to uncover some of the molecular history, which shaped the evolution of this group of plasmids. In particular, data obtained suggested a large amount of horizontal transfer and rearrangement of plasmid molecules between E. coli, Salmonella and Shigella. Moreover, interestingly some plasmids from Shigella share a common history with Salmonella and several hypothetical proteins form co-occurrence clusters, suggesting possible roles in plasmid maintenance and/or pathogenesis, which could be investigated by conventional genetic techniques.

The proposed method is general enough to be proposed as a new tool for comparative genomic analyses of bacteria and can work at least within the range of phylogenetic 


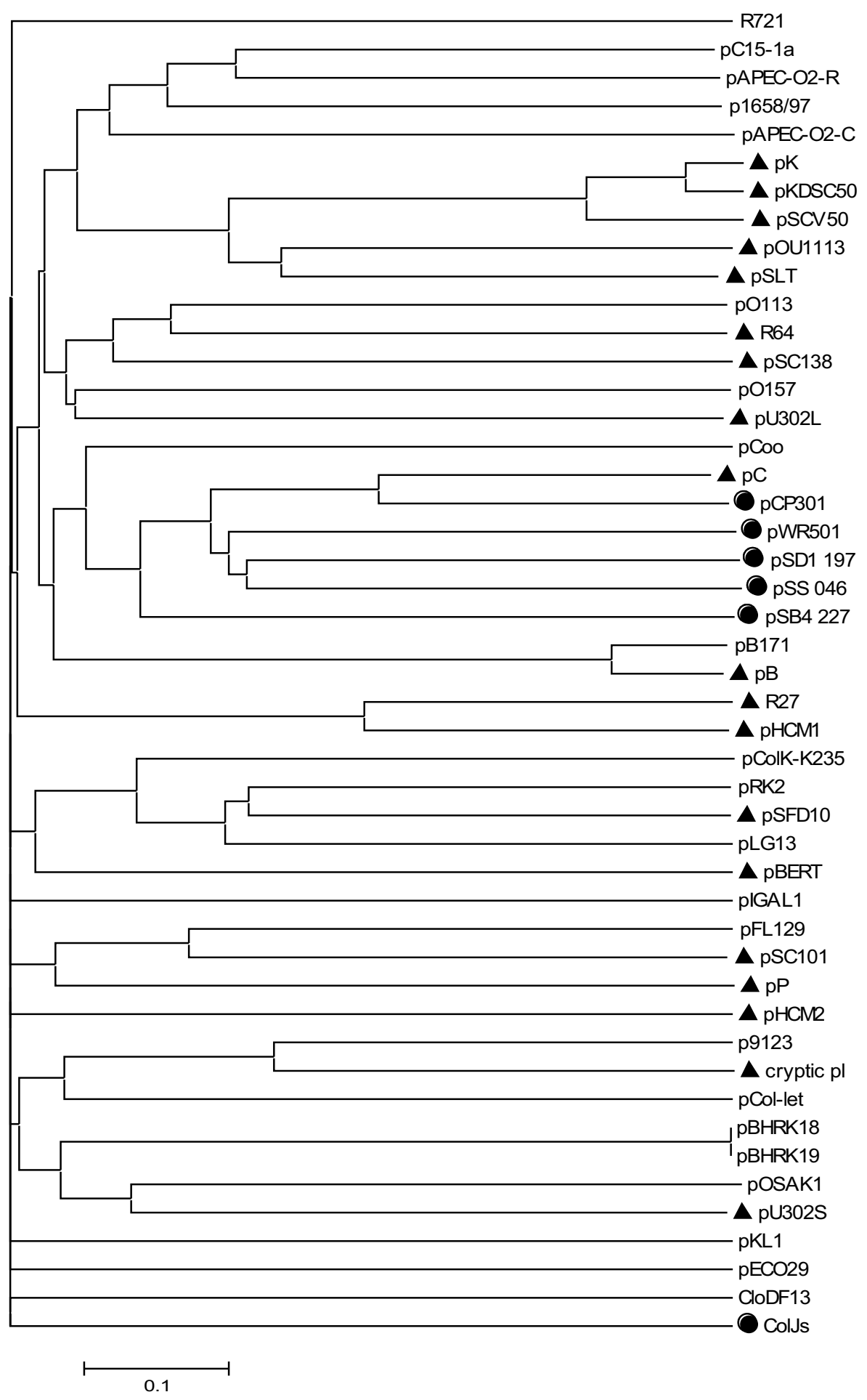

\section{Figure 4}

Neighbor-Joining dendrogram of the plasmids from phylogenetic profiling. Clustering of similarities in gene content of the plasmids obtained from their phylogenetic profile is reported (see text for details). Black circles or triangles before plasmid name refer to Shigella spp. or Salmonella spp. plasmids, respectively; Escherichia coli plasmids are not labeled. 




\section{Figure 5}

Neighbor-Joining dendrograms of protein co-occurrence pattern from phylogenetic profiling. Each cluster of this dendrogram includes those proteins that are commonly found together in the plasmids of the dataset reported in Table I. Each hypothetical protein is associated with the $\mathrm{Gl}$ of one representative of its corresponding protein cluster. 
distances enabling Blast to find homologs. For this reason, the B2N approach could help solving some questions linked to the presence of (few) well conserved functions within plasmid datasets from wide taxonomic ranges (e.g. functions related to transfer or replication). Moreover, possible applications of the method could include also chromosomal replicons, trying to depict histories of gene rearrangement and integration from plasmid to chromosomes and viceversa.

\section{Abbreviations}

B2N: Blast2Network; TTS: type III secretion system.

\section{Authors' contributions}

$\mathrm{MBr}$ participated in conceiving the idea, wrote the program and performed part of the analyses. AM, PL and RF participated in conceiving the idea. MF performed part of the analyses. MBa participated in discussing results. All authors contributed to draft the paper. All authors read and approved the final manuscript.

\section{Additional material}

\section{Additional file 1}

Figure $1 S$ - The functional activity of proteins from plasmid molecules present in GenBank database (as on March 2008). Histogram showing the putative roles of all the proteins (73909) encoded by the plasmids present in the NCBI repository. Each of the 73909 proteins was probed against the COG database http://www.ncbi.nih.gov/COG and its function was inferred according to the one assigned to the first BLAST hit of COG database. Data show that about $45 \%$ of the plasmid proteins deposited in the NCBI plasmids database have only a "general function" assignment or do not have any functional assignment at all.

Click here for file

[http://www.biomedcentral.com/content/supplementary/14712105-9-551-S1.pdf]

\section{Additional file 2}

Figure $2 S-H y p e r g r a p h$ of plasmid sequences. Similarity network showing the relationships existing among plasmids listed in Table 1, shown at two distinct thresholds. Differently from Figure 2, now each node represents a single plasmid and links the overall protein content shared among entire plasmids. In details, the size of nodes is proportional to the number of links possessed by a given plasmid, whereas the thickness of links was computed using p-values of the Jaccard distance calculated in phylogenetic profiling analysis (see text), hence accounting for an overall estimation of the shared proteins by each plasmids in respect to the others.

Click here for file

[http://www.biomedcentral.com/content/supplementary/1471-

2105-9-551-S2.pdf]

\section{Additional file 3}

Software availability, requirements and user manual. Project name: Blast2Network. Project home page: http://www. unifi.it/dblemm/CMpro$\underline{v-p-34 . h t m l}$. Operating system(s): Platform independent. Programming language: Java. Licence: GNU GPL. Any restrictions to use by non-academics: no restriction.

Click here for file

[http://www.biomedcentral.com/content/supplementary/14712105-9-551-S3.rar]

\section{Additional file 4}

Figure 3S - Phylogenetic profile with GI numbers of represented proteins as in Figure 5. Protein co-occurrence patterns (see text for details) including the GI numbers of those proteins taken as representatives of each single cluster of Figure 3.

Click here for file

[http://www.biomedcentral.com/content/supplementary/14712105-9-551-S4.pdf]

\section{Acknowledgements}

This work was supported by the Italian Ministry of Research, FISR founding "Soil sink". MBr was supported by a post doc fellowships of the University of Firenze.

\section{References}

I. Gilmour MW, Thomson NR, Sanders M, Parkhill J, Taylor DE: The complete nucleotide sequence of the resistance plasmid R478: defining the backbone components of incompatibility group $\mathbf{H}$ conjugative plasmids through comparative genomics. Plasmid 2004, 52:182-202.

2. Guerrero G, Peralta H, Aguilar A, Diaz R, Villalobos MA, MedranoSoto A, Mora J: Evolutionary, structural and functional relationships revealed by comparative analysis of syntenic genes in Rhizobiales. BMC Evol Biol 2005, 5:55.

3. Johnson TJ, Siek KE, Johnson SJ, Nolan LK: DNA sequence and comparative genomics of pAPEC-O2-R, an avian pathogenic Escherichia coli transmissible R plasmid. Antimicrob Agents Chemother 2005, 49:468I-4688.

4. Tauch A, Puhler A, Kalinowski J, Thierbach G: Plasmids in Corynebacterium glutamicum and their molecular classification by comparative genomics. J Biotechnol 2003, 104:27-40.

5. Kohiyama M, Hiraga S, Matic I, Radman M: Bacterial sex: playing voyeurs 50 years later. Science 2003, 301:802-803.

6. Thomas CM, Nielsen KM: Mechanisms of, and barriers to, horizontal gene transfer between bacteria. Nat Rev Microbiol 2005, 3:7||-72।.

7. Burrus $V$, Waldor MK: Shaping bacterial genomes with integrative and conjugative elements. Res Microbiol 2004, I 55:376-386.

8. Espinosa-Urgel M: Plant-associated Pseudomonas populations : molecular biology, DNA dynamics, and gene transfer. Plasmid 2004, 52: 139-150.

9. Dennis J]: The evolution of IncP catabolic plasmids. Curr Opin Biotechnol 2005, 16:291-298.

10. Karlin S, Burge C: Dinucleotide relative abundance extremes: a genomic signature. Trends Genet 1995, I I:283-290.

II. Fernández-López R, Garcillán-Barcia MP, Revilla C, Lázaro M, Vielva $L$, de la Cruz F: Dynamics of the IncW genetic backbone imply general trends in conjugative plasmid evolution. FEMS Microbiol Rev 2006, 30:942-966.

12. Pellegrini M, Marcotte EM, Thompson MJ, Eisenberg D, Yeates TO: Assigning protein functions by comparative genome analysis: protein phylogenetic profiles. Proc Natl Acad Sci USA 1999, 96:4285-4288.

13. Linton A, Hinton MH: Enterobacteriaceae associated with animals in health and disease. Soc Appl Bacteriol Symp Ser 1988, 17:7IS-85S.

14. Slater FR, Bailey MJ, Tett AJ, Turner SL: Progress towards understanding the fate of plasmids in bacterial communities. FEMS Microbiol Ecol 2008, 66:3-13.

15. Lavigne JP, Blanc-Potard AB: Molecular evolution of Salmonella enterica serovar Typhimurium and pathogenic Escherichia coli: from pathogenesis to therapeutics. Infect Genet Evol 2008, 8:217-226.

16. Altschul SF, Madden TL, Schaffer AA, Zhalg J, Zhalg Z, Miller W, Lipman DJ: Gapped BLAST and PSI-BLAST: a new generation of protein database search programs. Nucl Acids Res 1997, 25:3389-3402.

17. Sharp PM, Li WH: The codon Adaptation Index - a measure of directional synonymous codon usage bias, and its potential applications. Nucleic Acids Res 1987, I 5: I 28I-1295. 
18. Ramazzotti M, Brilli M, Fani R, Manao G, Degl'Innocenti D: The CAI Analyser Package: inferring gene expressivity from raw genomic data. In Silico Biol 2007, 7:507-526.

19. van Passel MW, Bart $A$, Luyf $A C$, van Kampen $A H$, Ende $A$ van der: Compositional discordance between prokaryotic plasmids and host chromosomes. BMC Genomics 2006, 7:26.

20. Larsen $\mathrm{MH}$, Figurski DH: Structure, expression, and regulation of the kilC operon of promiscuous IncP alpha plasmids. J Bacteriol 1994, 176:5022-5032.

21. Dionisio F, Matic I, Radman M, Rodrigues OR, Taddei F: Plasmids spread very fast in heterogeneous bacterial communities. Genetics 2002, 162:1525-1532.

22. Arthur DC, Ghetu AF, Gubbins MJ, Edwards RA, Frost LS, Glover JN: FinO is an RNA chaperone that facilitates sense-antisense RNA interactions. EMBO / 2003, 22:6346-6355.

23. Aguirre-Ramirez M, Ramirez-Santos J, Van Melderen L, GomezEichelmann MC: Expression of the F plasmid ccd toxin-antitoxin system in Escherichia coli cells under nutritional stress. Can J Microbiol 2006, 52:24-30.

24. Escobar-Paramo P, Giudicelli C, Parsot C, Denamur E: The evolutionary history of Shigella and enteroinvasive Escherichia coli revised. J Mol Evol 2003, 57:140-148.

25. Hartman AB, Essiet II, Isenbarger DW, Lindler LE: Epidemiology of tetracycline resistance determinants in Shigella spp. and enteroinvasive Escherichia coli: characterization and dissemination of tet(A)-I. J Clin Microbiol 2003, 4 I: I023-1032.

26. Boyd EF, Hartl DL: Recent horizontal transmission of plasmids between natural populations of Escherichia coli and Salmonella enterica. J Bacteriol 1997, I79: I622-I627.

27. Call DR, Kang MS, Daniels J, Besser TE: Assessing genetic diversity in plasmids from Escherichia coli and Salmonella enterica using a mixed-plasmid microarray. J Appl Microbiol 2006, 100: I5-28.

28. Thomas CM: Paradigms of plasmid organization. Mol Microbiol 2000, 37:485-49l.

29. Tian W, Skolnick J: How well is enzyme function conserved as a function ofpairwise sequence identity? I Mol Biol 2003, 333:863-882.

30. Friedberg I: Automated protein function prediction - the genomic challenge. Brief Bioinform 2006, 7:225-242.
Publish with Bio Med Central and every scientist can read your work free of charge

"BioMed Central will be the most significant development for disseminating the results of biomedical research in our lifetime. "

Sir Paul Nurse, Cancer Research UK

Your research papers will be:

- available free of charge to the entire biomedical community

- peer reviewed and published immediately upon acceptance

- cited in PubMed and archived on PubMed Central

- yours - you keep the copyright
BioMedcentral 